R. Gogsadze, A. Prangishvili, P. Kervalishvili, R. Chiqovani and V. Gogichaishvili

\title{
A boundary problem of micro- and nano-electronics
}

ABSTRACT. The boundary problem discussed concerns the energy state of an electron in a system of immovable electrically charged particles. The main principle followed was that the atoms of the substance (neutral and ionized) are not themselves systems and consist of discrete material particles (the Rutherford model). According to the principles of unified field theory, these atoms are localized unified fields (uninterrupted continua) located in some final volume, beyond the limits of which the parameters characterizing the material properties of these unified fields quickly diminish. In other words, the sources of electric field in the volume in which the investigated system is located are the immovable ionized entities and the mobile electrons. Their distributions define the spacial structure of the electric field in the volume, and the structure of the electric field determines the quantum state (distribution) of electrons in the system; i.e., these two aspects are interrelated. Here, the functional equations (Poisson and Schrödinger) of the electrons in the system are derived. These differential equations are formulated with real boundary conditions. An original method for the solution of the boundary problem for a particular case is proposed.

Nanotechnology Perceptions 12 (2016) 173-183

doi: 10.4024/N15GO15A.ntp.12.02 